\title{
PROTECTION BY WR-151327 AGAINST LATE-EFFECT DAMAGE FROM FISSION-SPECTRUM NEUTRONS
}

\author{
D. J. Grdina ${ }^{12}$, B. J. Wright', and B. A. Carnes \\ 'Biological and Medical Research Division \\ Argonne National Laboratory \\ Argonne, Illinois 60439 \\ ${ }^{2}$ Department of Radiation and Cellular Oncology \\ University of Chicago \\ Chicago, Illinois 60637
}
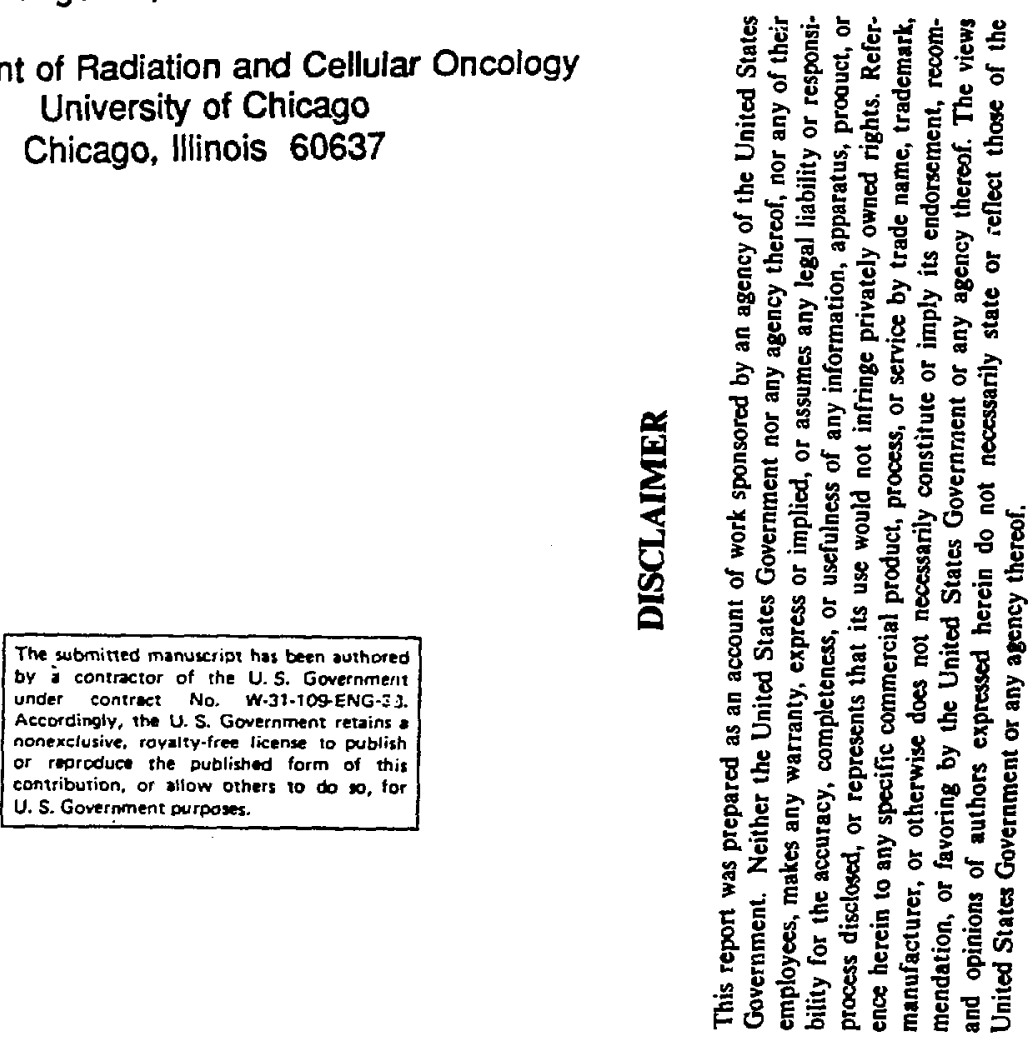

Keywords: Radiation protector, WR-151327, neutrons, late effects, life shortening 


\section{INTRODUCTION}

Considerable effort has been expended to develop chemical agents capable of modifying radiation-induced damage to biological systems (1). Most of this work has focused on maximizing the ability of these agents to protect against acute lethal events $(2,3)$. Maisin and others have demonstrated the ability of chemical agents to protect against radiation-induced late-effect damage in rodents (4-6). The endpoint studied was life shortening, a phenomenon mediated in large part by the onset of lethal radiationinduced tumors (7).

Recent reports have demonstrated that nonlethal genotoxic damage from either low- or high-LET radiations can be significantly reduced by aminothiol radioprotector chemicals $(8,9)$. Protection was observed even when the radioprotectors were administered up to three hours following radiation exposure. Under in vitro conditions, WR-1065, the free thiol of WR-2721, can protect against mutations in V79 cells at the hypoxanthine-quanine phosphoribosyl transferase (HGPRT) locus following exposure to either ${ }^{60} \mathrm{Co}$ gamma rays (8) or $0.85-\mathrm{MeV}$ fission-spectrum neutrons (9). These data demonstrated that radiation-induced genotoxic damage can be protected against by the administration of a radioprotector up to three hours following radiation exposure. When administered after irradiation, no protection against cytotoxicity was observed.

Work in our laboratory has focused on the characterization and application of aminothiol compounds as antigenotoxic agents. WR-1065 can protect against the clastogenic effects of neutrons and gamma rays $(10)$ and ${ }^{60} \mathrm{Co}$-induced transformation in C3H10T1/2 cells (11). WR-2721 protects neonatal rats against radiation-induced preneoplastic lesions (12). We have expanded our studies to determine whether the 
chemical radioprotector WR-151327 [S-3-(3-methylaminopropylamino) propylphosphorothiotic acid] can be used to protect mice against the life-shortening effects of fission-spectrum neutrons. This agent affords excellent protection against neutron-induced damage to the jejunum of mice (13), and it can be administered orally.

\section{MATERIALS AND METHODS}

The $\mathrm{B}_{6} \mathrm{CF}_{1}$, hybrid mouse (i.e., C57BL/6J ANL female $\times$ BALB/aJ ANL male) was used in this study. Animals were 110 days of age at the time of irradiation. Four hundred mice (two hundred of each sex) were randomly assigned to each of the two treatment groups. Animals were routinely housed five per cage, maintained in animal rooms of 200 cage capacity, and provided with access to food and water at all times. Temperature and humidity was controlled to $72 \pm 4^{\circ} \mathrm{F}$ and $50 \pm 10 \%$ relative humidity. A 12 -hour light/dark cycle was always used.

Irradiations were performed using the JANUS reactor (14). Neutron doses were 10 cGy whole body. Animals were individually placed in one-pint polyethylene containers for irradiation and then returned to their cages. Controls were sham-irradiated. All operational logistics and cage locations in the holding rooms were controlled by computer and randomized to avoid experimental biases due to human decision making. Death checks were performed daily, and all mice were examined to ascertain cause of death and other incidental findings.

WR-151327 was supplied by Col. David E. Davidson, Jr. A dose of $580 \mathrm{mg} / \mathrm{kg}$ was administered I.P. to animals 30 min prior to irradiation. 
All life-shortening data was subjected to rigorous statistical analysis. The productlimit method described by Kaplan and Meier (15) was used to estimate the survival distribution function for each group in the study. Males and females were analyzed separately, and all analyses were performed on the IBM 3033 at Argonne National Laboratory using the LIFETEST procedure in the SAS sottware package (16). Data are presented as cumulative survival curves for time intervals of 300 to 850 days and 850 to 1300 days following irradiation.

\section{RESULTS}

Animals injected I.P. with $580 \mathrm{mg} / \mathrm{kg}$ of WR-151327 and later exposed to JANUS fission-spectrum neutrons were afforded protection against the life-shortening effects of the radiation. A break point of 850 days post irradiation was arbitrarily chosen because it approximates the median survival time of neutron-exposed animals.

The magnitude and timing of protection depended on the sex of the animal. From 300 to 850 days following irradiation, no statistical differences were found between the cumulative survival curves of treated and control male animals $(P=0.30$; Fig. 1$)$. In contrast, female animals during this interval were protected (Fig. 2), as demonstrated by the longer survival times of the treated animals $(P=0.02)$.

Analysis of the cumulative survival curves between 850 and 1300 days showed a different relationship. For male animals, treatment with WR-151327 appeared to enhance survival beyond the 1000-day point (Fig. 3); a comparison of these curves yielded a $P$ value of 0.14 . The cumulative survival curve of the protected animals was shifted to longer survival times. No significant difference was observed for female animals 
(Fig. 4; $P=0.37$ ), but the cumulative survival curve for the WR-151327 exposed animals was shifted to shorter survival times.

\section{DISCUSSION}

Life shortening has been used as a measure of chronic radiation injury because it is an efficient integrator of cumulative multisystemic injury over a lifetime (7). In the $\mathrm{BCF}_{1}$ mouse system, $85 \%$ of the accelerated deaths due to radiation exposure are related to tumors (7). We have verified this finding in selected groups of animals by histopathological analysis following routine necropsy procedures. A detailed account of these data will be presented elsewhere.

We recently reported that the radioprotectors WR-2721 and WR-151327 can protect against neutron-induced life shortening in the B6CF $_{1}$ hybrid mouse system (17). A major mechanism of action implicated in these protective effects is free-radical scavenging $(18,19)$. However, neutrons are believed to cause injury directly, in contrast to low-LET photons, which act indirectly through free-radical formation. While the role of free-radical scavenging can not be ruled out as an important mechanism in the action of these radioprotectors, these agents are known to be effective in binding to DNA and stabilizing chromatin $(20,21)$, affecting DNA synthesis (22), and affecting cell cycle progression (23). Each of these properties can affect the subsequent expression of radiation-induced genotoxic damage.

We describe here differences in the radioprotective effect of WR-151327, depending on the sex of the animal and the post-irradiation time interval considered. The greatest effect in female animals is prior to 850 days post irradiation. The greater 
protection in male animals is seen during the time increment following 850 days after irradiation. While it is difficult at present to ascribe these effects to a particular model, it is suggestive that hormonal factors may play a role in aminothiol protection against radiation-induced life shortening and concomitant tumor induction in the $\mathrm{B} 6 \mathrm{CF}$, hybrid mouse system. With respect to subsequent tumor induction, our preliminary findings to be published elsewhere suggest that tumors of lymphoreticular origin are the class of tumors most affected by the administration of a radioprotector prior to irradiation. 


\section{ACKNOWLEDGMENTS}

This work was supported in part by the U.S. Department of Energy, Office of Health and Environmental Research, under Contract W-31-109-ENG-38, the Center of Radiation Therapy, and NIH/NCl Grant CA-37435. Radioprotector compounds were kindly supplied by Col. David E. Davidson, Jr., Director, Division of Experimental Therapeutics, Walter Reed Army Medical Center, Washington, DC, 20307. We acknowledge the assistance of P. Dale and J. Perrin. We thank G. Holmblad, H. Gaines, and $G$. Shirvin for performing the dosimetry and irradiations, and C. Fox for computer analysis of the data.

\section{REFERENCES}

1. Patt, H. M., Tyree, E. B., Straube, R. L., and Smith, D. E. Cysteine protection against x-irradiation. Science 110: 213-214, 1949.

2. Yuhas, J. M., and Storer, J. B. Differential chemoprotection of normal and malignant tissues. J. Natt. Cancer Inst. 42: 331-335, 1969 a.

3. Yuhas, J. M., and Storer, J. B. Chemoprotection against three modes of radiation death in the mouse. Int. J. Fadiat. Biol. 15: 233-237, 1969b.

4. Maisin, J. R., Mattelin, G., and Lambiet-Collier, M. Chemical protection against the long-term effects of a single whole-body exposure of mice to ionizing radiation, I. Life shortening. Radiat. Res. 71: 119-131, 1977.

5. Maisin, J. R., Decleve, A., Gerber, G. B., Mattelin, G., and Lambiet-Collier, M. Chemical protection against the long-term effects of a single whole-body exposure of mice to ionizing radiation, II. Causes of death. Radiat. Res. 74: 415-435, 1978. 
6. Maisin, J. R., Gerber, G. B., Lambiet-Collier, M., and Mattelin, G. Chemical protection against long-term effects of whole-body exposure of mice to ionizing radiation, III. Effects of fractionated exposure to C57BL mice. Radiat. Res. 82: $487-497,1980$.

7. Grahn, D., Thomson, J. F., Carnes, B. A., Williamson, F. S., and Lombard, L. S. Comparative biological effects of low dose, low dose-rate exposures to fission neutrons from the JANUS reactor or to Co-60 gamma rays. Nic. Sci. Appli. 2: 385-396, 1986.

8. Grdina, D. J., Nagy, B., Hill, C. K., Wells, R. L., and Peraino, C. The radioprotector WR-1065 reduces radiation-induced mutations at the hypoxanthineguanine phosphoribosyl transferase locus in V79 cells. Carcinogenesis 6: 929-931, 1985.

9. Grdina, D. J., Sigdestad, C. P., and Carnes, B. A. Protection by Wr-1065 and WR-151326 against fission-neutron-induced mutations at the HGPRT locus in V79 cells. Radiat. Res. 117: 500-510, 1989.

10. Schwartz, J. L., Giovanazzi, S. M., Karrison, T., Jones, C., and Grdina, D. J. 2 -[(aminopropyl) amino] ethanethiol-mediated reductions in ${ }^{60} \mathrm{Co}$ gamma-ray and fission-spectrum neutron-induced chromosome damage in V79 cells. Radiat. Res. 113: $145-154,1988$.

11. Hill, C. K., Nagy, B., Peraino, C., and Grdina, D. J. 2-[(aminopropyl) amino] ethanethiol (WR-1065) is anti-neoplastic and anti-mutagenic when given during ${ }^{60}$ Co gamma-ray irradiation. Carcinogenesis 7: 665-668, 1986. 
12. Grdina, D. J., Peraino, C., Cames, B. A., and Hill, C. K. Proiective effect at S-2(3-aminopropylamino) ethyl phosphorothioic acid against induction of altered hepatocyte foci in rats treated once with gamma-irradiation within one day after birth. Cancer Res. 45: 5379-5381, 1985.

13. Sigdestad, C. P., Grdina, D. J., Connor, A. M., and Hanson, W. R. A comparison of radioprotection from three neutron sources and ${ }^{60} \mathrm{Co}$ by WR-2721 and WR-151327. Radiat. Res. 106: 224-233, 1986.

14. Marshall, I. R., and Williamson, F. S. Microdosimetric spectra measurements of JANUS neutrons. Radiat. Protec. Dosim. 13: 111-11j, 1985.

15. Kaplan, E. L., and Meier, P. Nonparametric estimation from incomplete observations. J. Am. Stat. Assoc. 53: 457-481, 1958.

16. SAS Institute Inc., SAS User's Guide: Statistics, Version 5 Edition. SAS Inst. Inc., Cary, NC, 956 pp., 1985.

17. Grdina, D. J., Carnes, B. A., and Nagy, B. Protection by WR-2721 and WR-15127 against late effects of gamma-rays and neutrons. In: Advances in Space Research, XVIII COSPAR Meeting. Pergamon Press, Oxiord, England. In press, 1990.

18. Yuhas, J. M., Spellman, J. M., and Culo, F. The role of WP-2721 in radiotherapy and/or chemotherapy. Cancer Clin. Trials 3: 211-216, 1980.

19. Phillips, T. L. Rationale for initial clinical trials and future development of radioprotectors. Cancer Clin. Trials 3: 165-173, 1980.

20. Fahey, R. C. Protection by Thiols. Pharmac. Ther. 39: 101-108, 1988. 
21. Brown, P. E. Mechanism of action of aminothiol radioprotectors. Nature 213: 363-354, 1967.

22. LaSalle, M., and Billen, D. Inhibition of DNA synthesis in murine bone marrow cells by AET and cysteamine. Ann. New York Acad. Sci. 114: 622-628, 1964.

23. Grdina, D. J., and Nagy, B. The effects of 2- (aminopropyl) amino ethanethio! (WR-1065) on radiation-induced DNA damage and repair and cell progression in V79 cells. E. J. Cancer 54: 933-941, 1986. 


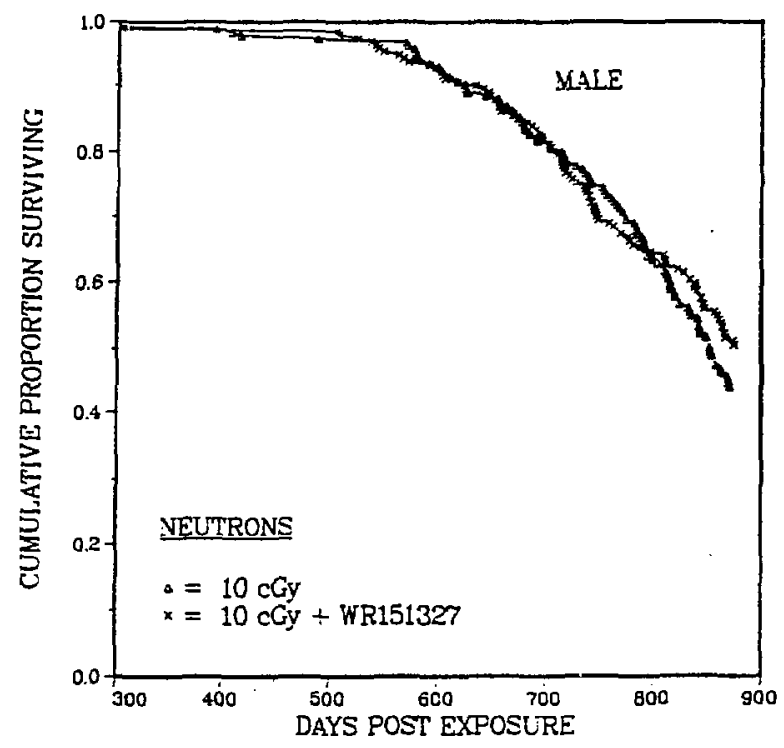

Fig. 1. Effect of WR-151327 on life shortening caused by fissionspectrum neutrons in male mice between 300 and 850 days following irradiation.

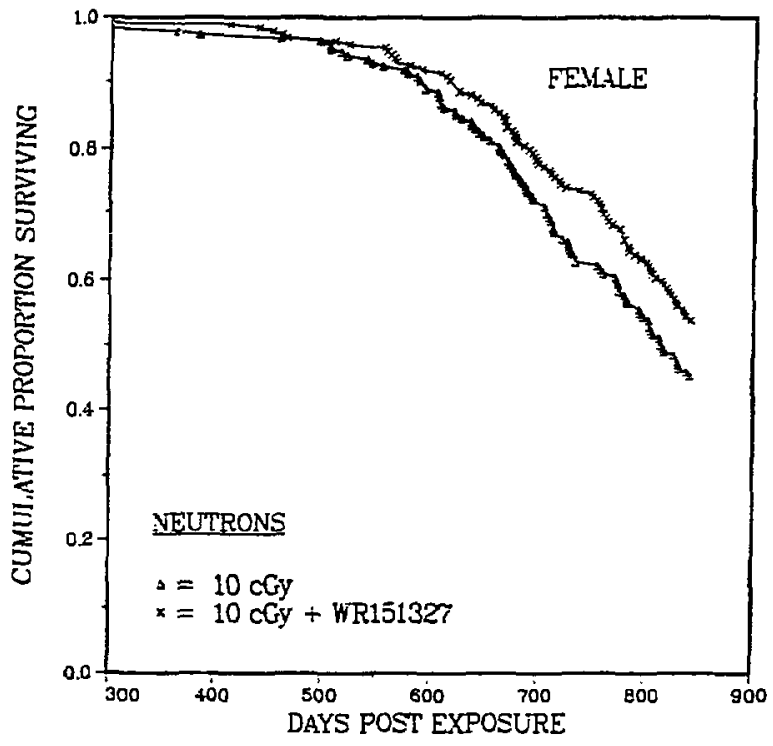

Fig. 2. Effect of WR-151327 on life shortening caused by fissionspectrum neutrons in female mice between 300 and 850 days following irradiation. 


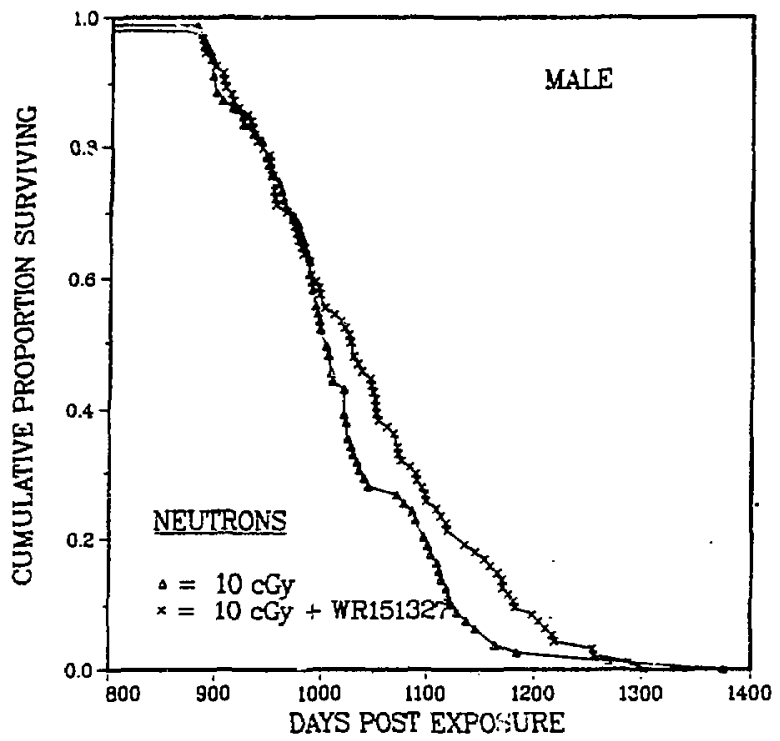

Fig. 3. Effect of WR-151327 on life shortening caused by fissionspectrum neutrons in male mice between 850 and 1300 days following irradiation.

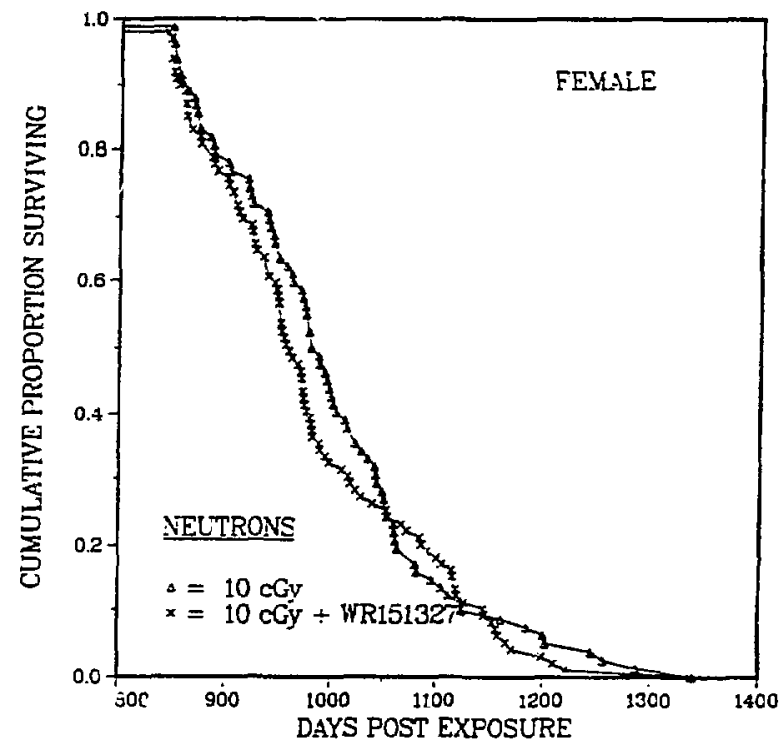

Fig. 4. Effect of WR-151327 on life shortening caused by fissionspectrum neutrons in female mice between 850 and 1300 days following irradiation. 